\author{
¿POLÍTICA SIN ÉTICA? \\ POLITICS WITHOUT ETHICS?
}

\author{
Jesús Ignacio Panedas Galindo \\ Universidad la Salle Pachuca
}

Nota sobre el autor

EI Mtro. Jesús Ignacio Panedas Galindo es licenciado en ciencias religiosas, licenciado en filosofía y maestría en filosofía. Actualmente es el coordinador de las materias de humanidades de la Universidad La Salle Pachuca.

Esta investigación fue financiada con recursos del autor.

Remita cualquier duda sobre este artículo al siguiente correo electrónico: jpanedas@lasallep.edu.mx

Recibido:1/1/2006

Aceptado:1/1/2006

Derechos de autor @ 2006 Universidad La Salle Pachuca - Todos los derechos reservados.

Condiciones de Uso: La reproducción total o parcial de la obra en soporte electrónico queda prohibida sin la autorización de la Universidad la Salle Pachuca. 


\section{¿PoLÍTICA SIN ÉTICA?}

\section{Resumen}

Jesús Ignacio Panedas Galindo*

Durante varios siglos se ha venido discutiendo la posible relación o no de la política con la ética. Niklas Luhmann es un autor reciente y que construye una novedosa teoría social. En este artículo hacemos un recorrido por lo esencial de su pensamiento para poder ubicar sus luces y sus sombras respecto al tema que analizamos.

\section{Abstract}

The relation between politics and ethics has been a point of discussion during severals centuries. Niklas Luhmann is a modern thinker who offers a recent social theory. In our work, we are analycing the essential part of his thought to be able to understand his strengths and weaknesses about our object.

\section{Presentación}

Durante la época de los clásicos griegos y del largo tiempo del predominio cristiano siempre se consideró que la política y la vida social de las ciudades debían estar hermanadas con la ética. Lo bueno para uno debía ser también lo bueno para todos. Maquiavelo, en los albores de la modernidad, rompió estos lazos y abiertamente reflexionó sobre la realidad política. "La política por la política" ha sido, desde entonces, la divisa de casi todos los gobernantes. Si eso es así para qué auto engañarnos. Desde el italiano hasta nuestros días la relación entre la teoría y la realidad del ejercicio político no han cambiado esencialmente.

Niklas Luhmann quizá sea el último gran teórico que ha defendido la necesaria separación entre estos dos términos. En las siguientes líneas intentaremos hacer un recorrido rápido por lo esencial del corpus luhmanniano. Como conclusión haremos una breve síntesis y crítica de su pensamiento e intentaremos ofrecer algún indicador para recuperar, en la práctica, la relación entre ética y política. 
* Licenciado en ciencias religiosas, licenciado en filosofía, maestría en filosofía. Actualmente es el coordinador de las materias de humanidades de la Universidad La Salle, campus Pachuca.

En el pensamiento luhmanniano se asienta que en los regímenes o sociedades anteriores a la actual sociedad de bienestar, existe un punto central, en torno al cual gira todo lo demás, llámese estado o política. Sin embargo, dentro del sistema funcional la sociedad es un todo compuesto por distintos subsistemas, entre los cuales podemos incluir el de la política.

Este subsistema tiene que interrelacionarse con otros tan importantes como él: religión, economía, educación, vida familiar, ética... Entre todos ellos tiene lugar una relación mutua con su entorno, formado por los subsistemas que le pueden afectar. De esta manera se explica que un mismo problema pueda tener distintos enfoques que deban ser resueltos por distintos subsistemas.

Por otra parte, y en contraposición al sistema clásico, es decir, evitando cualquier clase de centralismo, Luhmann integra al hombre en todos los subsistemas, pero sin terminar de incorporarse a ninguno de ellos en profundidad. El hombre, desde su lugar de entorno para cualquiera de los sistemas, puede comunicarse con ellos, pero no pertenece exclusivamente a ninguno (Luhmann 1990a, p.30).

Fundamentado en estos dos presupuestos, podemos avanzar en el análisis del desarrollo social de la sociedad del bienestar moderna. En este tipo de sociedad se ha ampliado desmesuradamente la capacidad de prestaciones comunicativas entre las distintas personas y sistemas; esto se traduce directamente en unas comunicaciones altamente especializadas y funcionales ${ }^{[11}$. Se ha llegado hasta estos niveles de comunicación principalmente por dos factores: diferenciación de una determinada clase superior ${ }^{[2]}$ y diferenciación de 
distintos centros de gravedad funcionales de la sociedad ${ }^{[3]}$. La complejidad da paso a la especialización y ambas necesitan de la funcionalidad para hacer posible la supervivencia del sistema. Veámoslo un poco más despacio.

\section{I) DIFERENCIA ENTRE SOCIEDAD CLÁSICA Y DEMOCRACIA ACTUAL}

La sociedad clásica era una sociedad claramente estratificada. Cada persona pertenecía a un estamento y le resultaba sumamente difícil salir de él y pasar a ser miembro de otro. La nobleza controlaba el poder político y el moral. Éste por venir su autoridad directamente desde Dios; y aquél, para dominar al hombre. El estado o la política era el centro de la sociedad monopolizado por una pequeña parte de la población. Todo giraba en torno a esta estructura vertical (Luhmann, 1990b, p. 127-128).

Por el contrario, la sociedad moderna o democracia es un sistema (Luhmann, 1994b, p. 151) sin portavoz y sin representación interna (Luhmann 1994a, p. 153). Por la indiferencia hacia otros sistemas y por la dinámica interna autónoma no necesita de portavoz ante nada ni nadie. De la misma manera, el criterio de funcionalidad reduce todo a esquema de utilidad, cada quien está en su lugar para hacer lo que sabe y debe hacer.

Se hace necesaria a esta altura la inclusión en nuestro estudio de la noción de diferenciación:

«La diferenciación sistémica no es más que una repetición de la constitución de sistemas en el interior de sistemas... De allí que el sistema de diferenciación sea un procedimiento de aumento de complejidad...» (Luhmann 1991, p. 40; Luhmann 1990b, p. 54).

Exactamente. La realidad moderna es compleja. Lo que antes era un todo ahora se subdivide en distintos campos de especialización. La diferenciación permite y realiza la existencia de distintos subsistemas individuales y 
especializados dentro del sistema. Cuanto mayor sea el número de subsistemas más compleja es la estructura del sistema global y más prolijas las interrelaciones entre ellos, con lo cual se aumenta la posibilidad de crecimiento en complejidad (Vallespin 1994, p. 14, Luhmann 1990b, p. 55).

Sin embargo, la diferenciación también puede ser contemplada como unidad («Unitas multiplex»). Por una parte, la diferencia mantiene unido lo diferente, por oposición a lo no diferente. La relación inevitable de los sistemas diferentes se puede interpretar como la unidad del sistema a partir del principio de construcción de su diferenciación. Por otra parte, mediante la diferenciación el sistema gana en sistematicidad. Crece en su propia identidad ${ }^{[4]} y$ en su diferenciarse consigo mismo[5].

Con todo lo dicho, podemos hablar de la sociedad democrática como un sistema funcional diferenciado de la política y de la moral. Entre la sociedad y la política o moral hay una relación como del todo a la parte. Estos últimos son unos subsistemas independientes, autónomos, autopoiéticos y no necesariamente interreferentes, que cumplen su función específica dentro de la sociedad sistémica. No pueden, en ningún momento y por ninguna circunstancia, ocupar el centro de algo que por definición no tiene centro ni vértice.

Actualmente no se puede centrar sobre la política una sociedad funcionalmente diferenciada sin destrozarla ${ }^{[6]}$.

\section{II) COMPLEJIDAD Y ESPECIALIZACIÓN}

La sociedad evoluciona hacia modelos más complejos. El de «complejidad» es un concepto que se relaciona con el de diferenciación, con el de la distinción entre sistema y entorno y con el de autorreferencia y autopóiesis. De esta manera unimos lo ya dicho y lo que se va a estudiar más abajo. 
«... por complejidad se entiende la existencia de un conjunto de posibilidades superior a las que de hecho pueden ser realizadas y exigen algún tipo de selección entre ellas; equivale a un "imperativo de selección" (Selektionszwang) entre el cúmulo de opciones que se abren a la acción» (Vallespín 1994, p. 14).

Luhmann constata la necesidad de un nuevo aparato teórico social que ayude a comprender y explicar las nuevas realidades en las que estamos viviendo. Para él es necesario hacer una revisión de todo el pensamiento anterior («llustración de la llustración») que ya ha llegado a su límite en la capacidad de describir la realidad (Izuzquiza 1990, p. 10; Luhmann 1993 p. 27-34; Luhmann 1991, p. 38).

Es por ello que la relación entre el todo y la parte de teorías anteriores se sustituye en la teoría sistemática por la relación entre el sistema y el entorno (Luhmann 1991, p. 39; Ludewig 1996, p. 112). Los sistemas no podrían existir sin su entorno, se mantienen «vivos» gracias al mantenimiento de las diferencias que lo distinguen y, al mismo tiempo, lo unen al entorno.

Esta relación va a ser siempre dinámica y va a permitir un grado progresivo de complejidad en ambos para dar solución a más cuestionamientos. Cada uno de los dos polos, sistema y entorno, tiene su propia identidad, mas su identidad se fundamenta en la interacción mutua de los dos (Luhmann 1993, p. 34-42) y, al mismo tiempo, en su diferenciación.

La complejidad de la sociedad implica que ya no exista una realidad dentro de ella que pueda coordinar la búsqueda de soluciones a todo, se impone la necesidad de diversificar las funciones. Conforme se ha ido dando la división de funciones se ha tenido que recurrir a la especialización (Luhmann 1990a, p. 3031). El creciente proceso de especialización ha desarrollado inmensamente el 
potencial de acción humano. La verdad científica, el dinero y el poder, organizado por la política y recortado por el derecho, son las guías que han de dirigir el desarrollo de la acción sistémica y funcional. Este proceso progresivo y autopoiético implica tres notas características:

* Por esta división especializada se han ido creando una multitud de sistemas funcionales que son sensibles a ciertos valores o cuestiones. Por otra parte, los datos que están fuera de su campo de interés son rechazados por la ignorancia o por considerarlos inútiles para las funciones de su sistema ${ }^{[71}$.

* La focalización en el mismo interés del sistema, el ensimismamiento ${ }^{[8]}$ en sus funciones, provoca en idéntica proporción una indiferencia respecto a lo que se refiere a otros sistemas.

* Las condiciones naturales del hombre favorecen esta especialización. Cada persona tiene habilidades distintas que debe favorecer.

Como conclusión a este apartado y teniendo en cuenta la progresiva especialización y funcionalidad, se puede afirmar que

«... una sociedad organizada en sistemas no dispone de ningún órgano central. Es una sociedad sin vértice ni centro» (Luhmann 1990a, p. 31 32 , nota 9).

\section{III) AUTORREFERENCIA (Del Campo 1987, p. 2060) Y AUTOPóIESIS}

Este concepto es otro de los fundamentos de la teoría sistémica que se complementa y necesita de los anteriores. Esta función nos describe la manera interna de cómo cada uno de los subsistemas se relacionan consigo mismos y cuál es la base de su relación con los entornos.

«Por autorreferente se entiende un sistema que produce y reproduce por sí mismo los elementos... de que está constituido»(Luhmann 1990a, p. 39-40). 
La autorreferencia es condición de las operaciones de un sistema. Éste está compuesto por distintos elementos que «ad intra» y por autocontacto van generando nuevos elementos ${ }^{[9]}$. El sistema, por tanto, desde su autorreferencialidad, es capaz desde sus propios elementos (Luhmann 1990b, p. 63) de crear nuevos componentes que lo constituyan. Es, en otras palabras, una autogeneración. Este proceso se supone imprescindiblemente para poder hablar de una relación del sistema con su entorno. Si no existe la autorreferencia o autocreación no se puede relacionar con lo que le rodea. $\mathrm{Y}$ el contacto con el entorno obliga al sistema a reaccionar (Luhmann 1990a, p. 42). Entre el sistema y su entorno hay una relación de reacción y de autogeneración. Esta idea de la autorreferencia es una consecuencia de la misma diferenciación de sistemas, de la evolución social a la que hemos llegado $\frac{110]}{}$.

La autorreferencia tal y como la propone nuestro autor supone la existencia de un sistema cerrado sobre sí mismo, Pero de igual manera, esa capacidad de autoobervación y autogeneración le permite abrirse al entorno, con lo cual también podemos hablar de un sistema abierto (Luhmann 1993, p. 40 y siguientes; Panedas 1998, 145-149). Este es un ejemplo más del poder creativo de la paradoja aparente de la funcionalidad de sistemas.

Con las investigaciones de Maturana y de Varela el concepto de autorreferencia recibe especial importancia bajo la denominación de autopóiesis. Podría definirse como referida

«... a sistemas dinámicos que pueden distinguirse como unidades en cuanto son una red de producción de componentes que constituyen la red de producción que los produce; especifican los límites de esta red y constituyen a esta red como unidad en su dominio de existencia» (Rodríguez 1994, p. 10; Luhmann 1994a, p. 145). 
De manera esquemática podríamos decir que un sistema autopoiético es cerrado operacionalmente y se encuentra estructuralmente determinado desde su propia condición; por otra parte, es circular y recursivo, es decir no hay causa ni efecto; está en constante contacto con su entorno adaptándose permanentemente al mismo (sistema abierto).

¿Cuál sería el chispazo que provoca esta dinámica imparable? Las distintas exigencias o necesidades que se le van presentando al sistema. En el caso concreto de la política, ella debe hacer frente a los nuevos requerimientos que se le van presentando por parte de su entorno (Luhmann 1990a, p. 102-103). Por una parte permanece la estructura básica inamovible (partidos políticos, derechos, organizaciones...); pero por otra, se incluye a la población en el ámbito de la política con el cúmulo de pretensiones legítimas que requieren respuesta $\stackrel{[111}{ }$.

La autorreferencia es ante todo un principio tautológico, es un continuo «ensimismamiento», un dedicarse la política a ella misma. Una dificultad o peligro del subsistema político es el del cortocircuito. Este fenómeno consiste en representarse a sí mismo en la crítica del otro, es decir, en una actividad política bipolar (gobierno/oposición), la crítica verificada de la oposición al gobierno implica que aquélla es competente para resolver lo criticado, lo cual no es claro ni evidente (Luhmann 1990b, p. 43; Seguí 1996, p. 45).

Otro problema de la autorreferencia es el del uso negativo. La sociedad es dinámica y vertiginosamente cambiante. La política debe entonces, decidir con más rapidez cada día (lo cual no es siempre adecuado) o en su defecto, no actuar hasta tener las condiciones necesarias (Luhmann 1994a, p. 152; Luhmann 1990b, p. 59.75; Luhmann 1991, p. 54).

\section{IV) ÉTICA}

Dentro del pensamiento luhmanniano, la moral es: 
«... el conjunto de condiciones de aprecio y desprecio objeto de la comunicación cuya validez es independiente de su concreción o abstracción, así como de que alcancen en cada caso un consenso fáctico o no» (Luhmann 1989, p. 50).

No se habla ya de la búsqueda de la felicidad o de la descripción de la perfección humana, sino que se trata fundamentalmente de una reflexión sobre principios y afirmaciones de carácter moral. La reflexión sobre la moral no puede salir del ámbito que propiamente le corresponde, ya que si no se impondría a otros sistemas distintos.

La moral entendida desde la perspectiva antigua ocupaba el centro de la sociedad, mas no admitía una reflexión sobre sí misma, sobre sus principios y sobre las distintas concepciones de lo moral. El problema de la moral era, pues, de autorreferencia. Podemos entender de esta manera las guerras promovidas por defender valores morales, persecuciones y revueltas por motivos religiosos... y es que la fuerza siempre acompañaba a la moral, ya que tiende a colocar al mismo nivel el aprecio propio y el desprecio ajeno, incita a compromisos de riesgo e interviene en las situaciones problemáticas (Luhmann 1989, 48-49).

Con el paso del tiempo la trascendencia moral en el pensamiento de cada época va desapareciendo y va perdiendo su centralidad (Luhmann 1994b, p. 149150). De tal manera que las preguntas más acuciantes de la sociedad se dirigen hacia las novedades de la política, de la economía, de la educación y del derecho. La moral se ubica en su lugar, como un subsistema más entre otros.

El peligro que se corre al querer entremezclar la moral con otros sistemas, por ejemplo el político, es el de entrar en una dinámica de palabrería y autojustificación degenerando la moral en palabrería y falsedad. Sería lo mismo que decir, que un argumento moral puede defenderse o atacarse según la 
circunstancia que más convenga. Sin embargo, Luhmann defiende la coherencia en el comportamiento y el intento de universalidad dentro de la moral (Luhmann 1994b, p.56). El que haya perdido su característica de centro dentro de la sociedad no implica que se pueda obrar aleatoriamente en cualquier situación.

Desde este contexto queremos destacar brevemente tres puntos importantes de Luhmann, que ya han sido insinuados, y que van a definir la relación entre ética y política dentro de su pensamiento.

* La individualidad subjetiva desaparece en la sociedad sistémica de Luhmann (Luhmann 1990b, p. 77; Izuzquiza 1990, p. 81-82). El hombre es un entorno de los subsistemas. Puede comunicarse con todos, pero no pertenece a ninguno. Puede protestar, hacer huelga o revoluciones (Navarrete 1994, p. 34), pero lo único que consigue es obligar al sistema a actuar. Nunca va a destruir la estructura que permanece intacta con sus características. No tenemos, por tanto, un sujeto moral como en otras teorías políticas (Luhmann 1990a, p. 35), únicamente un «sujeto funcional» (Vallespín 1994, p. 10).

* La política es un subsistema más dentro de la sociedad, no tiene porqué imponerse a nadie. Ella tiene en su interior su propia dinámica provocada por los requerimientos que se le hacen y su realidad con el entorno apropiado.

* La política, por ser otro subsistema autónomo, no necesita de ningún otro sistema para solucionar sus problemas (Luhmann 1996, p. 43-44; Salazar 1996, p. 48). La política no requiere más que de la política.

«... el sistema político no está para ser controlado de acuerdo a las bases del criterio moral; puede controlarse a sí mismo sólo políticamente... Parece que el sistema político, y lo mismo se puede aplicar a otros sistemas funcionales, establece por sí mismo la extensión y la forma por las cuales permite que la moralidad se vuelva relevante. Una ética política tendría, sobre todo, que tomar en cuenta la 
auto-dirección del sistema. Esto aún deja lugar para algunos florecimientos morales no controlados. La gente tiende a moralizar porque el contraste moral de bueno/malo les otorga la oportunidad de colocarse del lado de los ángeles, algo que la ética también tiene que reconocer» (Luhmann 1996, p. 47).

Llegamos, pues, a la conclusión con estas premisas, de que no es necesario que una norma ética afecte a la política (Luhmann 1996, p. 45. 44); es más, ésta debe ser totalmente amoral (Romero 1996, p. 53). La moral no puede pretender ocupar el centro del sistema, ni tan siquiera presentarse por encima del resto de los subsistemas. La función de la moral dentro de este pensamiento es integrar sistemas sociales en la sociedad. Ya no tiene la misión de permitir la entrada en sociedad o de provocar el rechazo (Luhmann 1989, p. 55).

CONCLUSIÓN

Veamos esquemáticamente las luces y las sombras del pensamiento luhmanniano.

\begin{tabular}{|c|}
\hline LUCES DE N. LUHMANN \\
\hline Fortaleza de la estructura \\
\hline Dinamismo \\
\hline Esfuerzo teórico novedoso \\
\hline Interdisciplinaridad \\
\hline
\end{tabular}

Es encomiable y respetable el esfuerzo que el autor alemán hace por ofrecer bases teóricas para poder observar de otra manera la sociedad. Es importante su empeño en destacar una estructura en constante desarrollo que permite una interminable interrelación entre los distintos subsistemas (Cortina 1994, p. 73). No se habla de algo informe que no consta de soporte ni, por el contrario, de una estructura rígida que nunca se somete al cambio. Esta es la base para trabajar 
sobre una interdisciplinaridad, en la estructura sistémica tienen cabida todas las ciencias.

\begin{tabular}{|c|}
\hline SOMBRAS DE N. LUHMANN \\
\hline Deshumanización de la sociedad \\
\hline Absolutización del dinamismo \\
\hline Formalismo \\
\hline Imposición teórica sobre la realidad \\
\hline
\end{tabular}

Lo que ya no parece tan aceptable es la desubicación de la persona humana hasta el entorno del sistema. El empeño teórico lo lleva en ocasiones a forzar la realidad o a no querer verla desde su objetividad (Solares 1996, p. 95). Por otro lado, el dinamismo, que era una virtud en su pensamiento puede ser una dificultad cuando se llega al extremo de reducir todo a dinamismo, a cambio, a movimiento.

El paradigma social que Luhmann propone es un modelo formal, que en el mejor de los casos, es factible en los países ya muy estructurados en donde la conciencia social del entramado político se reconoce perteneciente a un complejo mayor que él, la misma sociedad. Si añadiéramos una pizca de sorna a las posibilidades, podríamos decir que este esquema conceptual propuesto por Luhmann sería más propio de las ficciones futuristas de ciudades hipermodernas en las que se ausenta el conflicto, la violencia y, en definitiva, cualquier efecto de la acción libre del hombre.

Bueno sería que las sociedades fueran avanzando hacia las categorías operativas que el autor alemán propone. Tiempo antes que él, ya Hegel proponía la sociedad alemana de su tiempo como la mejor expresión del desarrollo del Espíritu humano. No cabía imaginarse más perfección ni un Estado más ordenado. Estas ilusiones darán remotamente como resultado la creencia que en la Alemania del siglo XX acogió el pensamiento y la acción más cruel conocida 
contra el ser humano (Klemperer 2002, 311-323). La maquinaria fría, perfectamente engrasada, del Estado se encauzó abiertamente al genocidio de inocentes. La historia nos deja muy en claro que esta perspectiva tiene sus peligros.

La realidad se impone obviamente y nos indica que esta sociedad utópica no existe en lo que sabemos. De lo que todos tenemos conocimiento y experiencia es de las limitantes particulares de cada sociedad y de cada gobierno en su ejercicio cotidiano. En ellas se plantea la tarea, que parece imposible (Cortina 1998, 21-24), de medir las impunidad de la política desde criterios valorales y éticos. La desconfianza progresiva en los políticos, en los periodistas, en los jueces y en las instituciones pudiera ser un llamado a la desilusión. Sin embargo, esta postura tanto en las personas como en las organizaciones, en lo privado como en lo público, debiera impeler más bien a un compromiso permanente de participación para no dejar toda la labor a los organismos públicos ni a la buena voluntad de alguien.

La conciencia de sabernos imperfectos y de reconocer limitadas a nuestras instituciones es la fuerza que nos obliga a compaginar el ejercicio funcional de lo político con la intención de cuidarnos y crecer en nuestra humanidad. De esto último trata la ética, de generar las posibilidades que nos permitan ser más personas, más humanos; de apropiarnos de nuestras propias posibilidades (Cortina 1998, 25-27).

Tanto la perfección sistémica como el derrotismo fatal son motivos de inoperancia y permanencia de lo establecido. El reconocimiento realista de las limitaciones y deficiencias se convierte en principio activador y causa de unificación entre la ética y el pragmatismo político. De esta manera nos abrimos al futuro, a los proyectos, a la ilusión, al compromiso participativo, a mejorarnos, a 
ser más justos, a vivir mejor. De esto debería hablarse cuando se discute sobre la vivencia del estado de bienestar. De esto debería hablarse cuando nos ocupamos de la situación social y personal del mexicano.

El idealismo formal de Luhmann, la deshumanización de su aparato teórico, más el reconocimiento de la realidad social y política de México nos llevan de la mano al convencimiento de dos principios fundamentales que pueden transformar nuestra realidad: el primero de ellos, reconocer la validez del principio funcional y siempre activo de Luhmann respecto de la sociedad; el segundo, la perentoria necesidad de convertir nuestra cultura en promotora de personas. En este segundo principio deberá incluirse la dimensión ética.

Funcionalidad y humanismo serían los dos grandes bastones con los que nuestra sociedad actual caminaría hacia un mejor futuro. Es por eso, que los dos polos (política y ética) de nuestro ensayo se necesitan hoy en México. No es suficiente conocer e intentar aplicar un molde extraño a nuestro espacio cultural (Ramos 1964, p. XX), es necesario poder tomar lo apropiado para construir nuestro propio camino hacia el porvenir mexicano.

\section{Referencias}

Adela CortinA, Hasta un pueblo de demonios. Ética pública y sociedad, Ed. Taurus, Madrid 1998.

Darío Rodríguez MANSILLA (1994), «Teoría de los sistemas organizacionales», en Sociología y política, II.

Fernando VALLESPíN (1994), «Introducción», en ID., Teoría política en el Estado de Bienestar, Madrid).

Ignacio IZUZQUIZA (1990)., La sociedad sin hombres. Niklas Luhmann o la teoría del escándalo, Ed. Anthropos, Barcelona. 
Javier TORRES NAFARRETE (1994), «La perspectiva Luhmaniana de la sociedad», en Sociología y política II.

Jesús Ignacio PANEDAS, ¿Es posible una relación entre ética y política?, en Mayéutica 24 (1998).

Jorge JAVIeR Romero (1996), «¿Cuál moral?» en Nexos XIX.

Kurt LUDEWIG (1996), Terapia sistémica. Bases de teoría y práctica clínicas, Ed. Herder, Barcelona.

Luis SALAZAR C. (1996), «El poder y la legalidad» en Nexos XIX.

Luis Seguí (1996), «Justiciables, justicieros y ajusticiados», en Nexos XIX.

Nicolás Maquiavelo (51997), El Príncipe. La Mandrágora, Ed. Cátedra, Madrid

Niklas LUHMANN (1989), «La moral social y su reflexión ética», en X. PAlAcios-F.. JaRAutA (eds.), Razón, ética y política. El conflicto de las sociedades modernas, Ed. Anthropos, Barcelona.

Niklas Luhmann (1990a), Political Theory in the Welfare State, Ed. Walter de Gruyter, Berlin-New York.

Niklas LUHMANN (1990b), Sociedad y sistema: la ambición de la teoría, Ed. Paidós, Barcelona.

Niklas LUHMANN (1991), Sistemas sociales. Lineamientos para una teoría general, Alianza Editorial, México.

Niklas LuHMAnN (1994a), "Complejidad y sistema social», en César CANSINOVíctor Alarcón Olguín, La filosofía Política de fin de siglo, Ed. UIA-Triana, México.

Niklas LuHmAnn (1994b), Teoría política en el Estado de bienestar, Alianza editorial, Madrid.

Niklas LUHMANN (1996), «Políticos, honestidad y la alta amoralidad de la política», en Nexos XIX.

Niklas LuHmann-Raffaele De Georgl (1993), Teoría de la sociedad, Ed. Universidad de Guadalajara-Universidad Iberoamericana-Instituto Tecnológico y de Estudios de Occidente, Guadalajara.

Salustiano DEL CAMPO (dir.) (1987), "Sociedad», en Diccionario Unesco de Ciencias Sociales IV, Ed. Planeta, Barcelona. 


\section{Samuel RAmos (1964), «La filosofía de Antonio Caso», en Antonio CASO, Antología filosófica, SEP, México. \\ V. KLemPerer, LTI. La lengua del Tercer Reich. Apuntes de un filólogo, Ed. Minúscula, Barcelona 2002.}

[1]Estos dos puntos, especialización y funcionalidad, son los que caracterizan de forma positiva a la sociedad de bienestar.

[2]Estas clases o estratos sociales gozaban de unas estrechas líneas de comunicación internas que han sido ampliadas y aplicadas a la globalidad del sistema.

[3]Estas dos diferenciaciones son las que componen la descripción «negativa» de la nueva sociedad. La universalización de ellas dan como resultado la sociedad de bienestar. Desde estas ideas podemos enlazar lógicamente con el ya mencionado de «especialización».

[4]Al reaccionar el sistema desde sí mismo a los influjos externos se está haciendo más «sí mismo»; aumenta su particularidad y su capacidad de enfrentar nuevos estímulos desde sus propias características.

[5]Cada vez que se hace más idéntico a sí mismo el sistema está elaborando nuevos elementos que le van a permitir seguir creando mayor complejidad desde su propia estructura, lo cual concluye en una diferenciación progresiva y dinámica respecto a su estado actual. Por eso digo "consigo mismo», porque se diferencia desde sus propias estructuras. Los elementos están inseparablemente unidos a la relación. Así como sistema y entorno conforman un código binario, de la misma manera no puede existir un elemento sin hacer relación a algo y no puede existir una relación que no haga mención a los elementos.

[6]La diferenciación de funciones existente en la sociedad, impide cualquier tipo de centralismo. Por otra parte, la relación sistema-entorno es tan dinámica y cambiante que la funcionalidad de los distintos subsistemas va variando por esta mutua influencia. La fuerza centrípeta de estos sistemas, originada entre las pretensiones y las soluciones, evitan se centren en nada que no sean ellos mismos.

[7]La apertura, pues, a una universalidad globalizada queda en entredicho. La función misma del sistema determina desde su interior el margen de interés al que se puede aspirar. La propia 
constitución del sistema limita las relaciones que éste puede tener con otros subsistemas determinados.

[8] Uso esta palabra sin ninguna connotación filosófica clásica. La entiendo como el progresivo centrarse en el ámbito exclusivo de un mismo sistema. Por este contenido no se puede deducir un proceso de conciencia o autoconocimiento. Hablamos normalmente en sentido funcional.

[9]Desde la idea de la autorreferencia llega Luhmann a la conclusión de que la decisión individual sólo puede tener sentido en conexión con el peso de las decisiones encadenadas dentro del sistema. Ello es, junto con la expulsión de la persona humana al entorno, dos limitaciones substanciales a la capacidad ética de la política y de la sociedad.

[10]Algunos autores critican la esterilidad y circularidad de esta explicación sistémica. Pero ello no es más que una adecuación global e histórica a las exigencias que se han ido presentando. El concepto, pues, tiene su relación con el de diferenciación funcional al que complementa.

[111Matizamos que la participación de la población no es vista desde el punto de vista del individuo, sino como procesos de comunicación política, a través de los cuales, la política se autosensibiliza. Lo importante es el intercambio de comunicación y la operatividad. No hay relación personal, sino funcional. Cuando se utiliza la palabra comunicación siempre va a ser sobre estas bases pragmáticas, no desde la interpersonalidad subjetiva. 